EFI-93-30

hep-th/9306013

\title{
Two Dimensional QCD coupled to Adjoint Matter and String Theory
}

\author{
David Kutasov \\ Enrico Fermi Institute \\ and Department of Physics \\ University of Chicago \\ Chicago, IL60637, USA
}

\begin{abstract}
We study $2 d$ QCD coupled to fermions in the adjoint representation of the gauge group $S U(N)$ at large $N$, and its relation to string theory. It is shown that the model undergoes a deconfinement transition at a finite temperature (analogous to the Hagedorn transition in string theory), with certain winding modes in the Euclidean time direction turning tachyonic at high temperature. The theory is supersymmetric for a certain ratio of quark mass and gauge coupling. For other values of that ratio, supersymmetry is softly broken. The spectrum of bound states contains an infinite number of approximately linear Regge trajectories, approaching at large mass $M, \alpha^{\prime} M^{2}=\sum_{i} i l_{i}\left(l_{i} \in \mathbf{Z}_{+}\right)$. Thus, the theory exhibits an exponentially growing density of bosonic and fermionic states at high energy. We discuss these results in light of string expectations.
\end{abstract}

$6 / 93$ 


\section{Introduction}

G. 't Hooft's suggestion [1] to generalize the gauge group of QCD from $S U(3)$ to $S U(N)$ and study the theory in a $1 / N$ expansion, is widely believed to be the most promising approach for obtaining quantitative analytical insight into the physics of confining gauge theory. The (known) properties of mesons in large $N$ gauge theory are in qualitative agreement with those observed in the real world (see e.g. [2]); yet, the large $N$ theory is expected to be significantly simpler than real QCD, essentially because the bound states become free as $N \rightarrow \infty$ (with interactions of order $1 / N$ ). The results of [1] and other arguments (see e.g. [3]) point to a possible relation of the $1 / N$ expansion of gauge theory to the topological expansion of some string theory, with $1 / N$ playing the role of the string coupling $g_{\mathrm{st}}$. Over the years there have been many attempts to make the correspondence more precise (see [4] for a recent review), but so far no concrete models have arisen. In fact, with an improvement of the understanding of unified string theory, it has been realized that there are some qualitative difficulties with a string description of QCD (in a $1 / N$ expansion):

1) All known string theories describe in one way or another space-time gravity. There seems to be no trace of that in QCD目.

2) There is a very general relation (to be elaborated on below) in string theory between the number of physical states and infrared stability [6]. That relation seems, in general, to be violated by gauge theories.

At the present state of the subject it is difficult to address these and other issues regarding large $N$ gauge theories in four dimensions. The purpose of this note is to discuss the situation in two dimensional gauge theory, with the hope of generating new ideas applicable in four dimensions. The choice of two dimensions is of course due to tractability, but we would like to retain as many of the essential features of $4 d$ gauge theory as possible. In particular, it seems that the number of degrees of freedom (and the related issue of the existence of deconfinement/Hagedorn transitions) plays an important role in string-like models. In gauge theory, much of the non-trivial structure of [1] seems to be related to the existence of propagating fields in the adjoint representation of $S U(N)$, the transverse gluons. Thus, pure $2 d \mathrm{QCD}$, which has been recently discussed from a string theory point of view [7] is inappropriate for our purposes, having no field theoretic degrees

1 Although perhaps it is not completely ruled out. Space-time gravity does arise in certain 0,1 dimensional large $N$ matrix models that received a lot of attention recently [5]. 
of freedom (and no deconfinement transition). The 't Hooft model [8] of complex fermions in the fundamental representation of $S U(N)$ exhibits a single asymptotically linear meson "Regge trajectory", and it should be interesting to reformulate it as a (perhaps open and closed) string theory. It too does not exhibit a deconfinement transition. We will concentrate here on the theory of (Majorana Weyl) fermions in the adjoint representation of the gauge group, described by the Lagrangian,

$$
\mathcal{L}=\frac{1}{g^{2}} F_{\mu \nu}^{2}+\bar{\psi} \gamma^{\mu} D_{\mu} \psi+m \bar{\psi} \psi
$$

where, as usual, $F_{\mu \nu}=\partial_{[\mu} A_{\nu]}+\left[A_{\mu}, A_{\nu}\right], D_{\mu} \psi=\partial_{\mu} \psi+i\left[A_{\mu}, \psi\right], \psi_{a b}$ is a traceless hermitian anticommuting matrix, $m$ is the (bare) fermion mass and $g$ the gauge coupling 2 . The hope is that the adjoint fermions mimick the effect of transverse gluons and make the theory non-trivial. The main reason for our interest in (1.1) is that (as we'll see below) this theory exhibits an infinite number of asymptotically linear "Regge trajectories", and undergoes a deconfinement transition at a finite temperature much like (what is expected in) four dimensional gauge theories. Nevertheless, it is more amenable to quantitative analysis, which can perhaps shed light on the relation to string theory and teach us other valuable lessons about higher dimensional gauge theory.

In section 2 we discuss a method, due to J. Polchinski, to study the stability of the confining phase at high temperature in a language familiar in string theory - in terms of masses of certain winding modes around the (Euclidean) time direction. Applying these ideas to $2 d$ QCD provides a qualitative guide to the different theories. We show that models with adjoint matter lose confinement at a finite temperature, unlike those with fundamental matter or no matter at all. This implies a rich spectrum of bound states.

In section 3 we turn to a more detailed analysis of QCD coupled to quarks in the adjoint representation of $S U(N)$. We describe the light cone quantization of the model, and show that it exhibits an in general explicitly broken supersymmetry, which is restored for a certain ratio of quark mass to gauge coupling.

In section 4 we discuss the spectrum of heavy bound states of adjoint quarks. We find a spectrum of masses which looks quite "stringy", consisting of an infinite number of asymptotically linear Regge trajectories. The density of single particle states increases exponentially with energy.

Section 5 contains some remarks on the topological space-time theory obtained in the far infrared, and a proof that supersymmetry is not spontaneously broken in this model. We conclude in section 6 with some comments about the results and their relation to string theory.

2 This model has been recently studied in [9]. 


\section{The Hagedorn transition in $2 d$ QCD}

At finite temperature one studies the theory (1.1) on a Euclidean cylinder $R \times S^{1}$; the $S^{1}$ describes Euclidean time which is periodic: $\tau \sim \tau+\beta$ ( $\beta$ is the inverse temperature), with boundary conditions for the gauge field and fermions (see e.g. [10]):

$$
A^{\mu}(\beta, x)=A^{\mu}(0, x) ; \quad \psi(\beta, x)=-\psi(0, x)
$$

The phase structure is analyzed in terms of the behavior of Wilson loops winding around the (compact, Euclidean) time direction:

$$
\rho_{k}(x)=\frac{1}{N} \operatorname{Tr} P \exp \left[i \int_{0}^{k \beta} d \tau A_{0}(\tau, x)\right]
$$

$P$ denotes path ordering; $k$ is the winding number of $\rho_{k}$. In the confining phase, correlators of $\rho_{k}(x)$ fall off exponentially at large $x$ :

$$
\left\langle\rho_{k}(x) \rho_{-k}(0)\right\rangle \sim \exp \left[-M_{k}(\beta) x\right]
$$

At low temperatures $(\beta \rightarrow \infty)$ one expects an area law, $M_{k}^{2}(\beta) \simeq k^{2} \beta^{2}$. As the temperature is raised, $M_{k}^{2}(\beta)$ decrease, until at some $\beta_{c}$ the first of the winding modes becomes tachyonic; equivalently, the effective string tension vanishes (the transition may actually be first order [11] and so occur before $\beta_{c}$ is reached). Above the critical temperature confinement is lost.

This description of the deconfinement transition is reminiscent of the standard picture of the Hagedorn transition in string theory (see e.g. [11]). The similarity has motivated a beautiful suggestion by J. Polchinski [12] of a way to probe the stability of the confining phase in gauge theory by calculating $M_{k}(\beta)$ (2.3) at high temperature, where perturbative techniques are reliable. We will next outline the application of these ideas to $2 d$ QCD (a more detailed account will appear elsewhere [13]).

At finite temperature one may choose an analog of the $A_{0}=0$ gauge:

$$
A_{a b}^{0}(\tau, x)=\frac{\theta_{a}(x)}{\beta} \delta_{a b}
$$

From (2.2), (2.4) it is clear that $\theta_{a}$ are periodic with period $2 \pi$. The gauge field $A_{a b}^{1}$ appears quadratically in the action (1.1) and can be integrated out, giving rise目 to an effective action for $\theta, \psi$ :

$$
\mathcal{L}_{\text {eff }}=\frac{1}{g^{2} \beta^{2}}\left(\theta_{a}^{\prime}\right)^{2}+\bar{\psi} \gamma^{\mu} D_{\mu}^{(0)} \psi+m \bar{\psi} \psi+g^{2} J_{1} \Omega^{-2} J_{1}
$$

3 In the process of integrating out $A_{1}$ one also finds a $\log \operatorname{det}\left(\partial_{\tau}^{2}+\theta^{2}\right)$, which can be shown to be unimportnat for what follows. 
where the following notation has been used: $\partial_{x}$ is denoted by a prime (here and below), $D^{(0)}$ is the covariant derivative (1.1) with respect to the gauge field $A_{1}^{a b}=0, A_{0}^{a b}$ given by (2.4). $J_{1}$ is the space component of the fermion $S U(N)$ current, $J_{\mu}^{a b}=\bar{\psi}^{a c} \gamma_{\mu} \psi^{c b}$, and $\Omega_{a b}$ is the operator:

$$
\Omega_{a b}=i \delta_{a b} \partial_{\tau}+\frac{\theta_{a b}}{\beta} ; \quad \theta_{a b} \equiv \theta_{a}-\theta_{b}
$$

To determine the behavior of $\rho_{k}$ (2.2), (2.3) we have to integrate out $\psi$ and study the resulting action for $\theta_{a}$. This is particularly easy to do at high temperature, since then the effective super-renormalizable couplings $g, m$ are small and it suffices to sum one loop diagrams. Thus we drop the last term in (2.5) (we'll keep the mass term for the time being since its presence will not complicate the calculation and allow us to make a few points later), and integrate out $\psi$. The action for $\theta$ we obtain is:

$$
\mathcal{S}_{\theta}=\frac{1}{g^{2} \beta} \int d x\left(\theta_{a}^{\prime}\right)^{2}+V\left(\theta_{a b}\right)
$$

where $V\left(\theta_{a b}\right)=-\log \operatorname{det}\left[\left(D^{(0)}\right)^{2}+m^{2}\right]$ and $\theta_{a b}$ is as in (2.6). The potential $V$ is easily evaluated (for slowly varying $\theta_{a b}$, which is the case appropriate for high temperature) following [10] (see also [14]). Dropping a $\beta$ independent constant corresponding to the zero temperature determinant, one finds:

$$
V(\theta)=\frac{\beta L}{2 \pi} \sum_{k=1}^{\infty}(-)^{k} \int_{0}^{\infty} \frac{d s}{s^{2}} \exp \left(-\frac{k^{2} \beta^{2}}{4 s}-s m^{2}\right) \cos k \theta
$$

$L$ is the length of space. In the UV limit $\beta \rightarrow 0$ the mass $m$ is irrelevant. Indeed, rescaling $s \rightarrow s \beta^{2} k^{2} / 4$ in (2.8) we see that the effective mass is $m \beta k / 2$, as mentioned above. Thus, as $\beta \rightarrow 0$ the effective action for $\theta$ approaches:

$$
\mathcal{S}_{\theta}=\int d x\left[\frac{1}{g^{2} \beta}\left(\theta_{a}^{\prime}\right)^{2}+\frac{2}{\pi} \sum_{a, b=1}^{N} \sum_{k=1}^{\infty}(-)^{k} \frac{1}{k^{2} \beta} \cos k \theta_{a b}\right]
$$

Of course, the last term in (2.5) which we neglected (as well as the mass and other terms) will induce corrections which become significant at finite $\beta$.

At large $N$ it is convenient to describe the dynamics of $\theta_{a}$ (or $\rho_{k}(2.2)$ ) by the density:

$$
\rho(\theta, x)=\frac{1}{N} \sum_{a} \delta\left(\theta-\theta_{a}(x)\right)
$$


In terms of $\rho$, the action (2.9) takes the form:

$$
\mathcal{S}_{\theta}=\frac{N^{2}}{\beta g^{2} N} \int d x \int d \theta \frac{1}{\rho(\theta, x)}\left(\partial_{\theta}^{-1} \rho^{\prime}\right)^{2}+N^{2} \int d x \iint d \theta_{1} d \theta_{2} \rho\left(\theta_{1}\right) \rho\left(\theta_{2}\right) V\left(\theta_{12}\right) / L
$$

To study the stability of the confining phase at $\beta \rightarrow 0$ one now assumes [12] that the eigenvalue distribution is approximately uniform, i.e.:

$$
\rho_{\mathrm{cl}}=\frac{1}{2 \pi}
$$

Expanding about $\rho_{\mathrm{cl}}$ :

$$
\rho(\theta, x)=\frac{1}{2 \pi}\left[1+\sum_{k \neq 0} \rho_{k}(x) e^{-i k \theta}\right]
$$

should show whether small fluctuations destabilize (2.12). As is suggested by the notation, $\rho_{k}$ in $(2.13)$ are the same as in (2.2), so we are actually calculating $M_{k}(\beta \rightarrow 0)(2.3)$. Plugging (2.13) in (2.11) and keeping only quadratic terms we find:

$$
\mathcal{S}_{\text {lin }}=N^{2} \sum_{k \neq 0} \int d x\left(\frac{1}{\beta g^{2} N k^{2}}\left(\rho_{k}^{\prime}\right)^{2}+\frac{2}{\pi}(-)^{k} \frac{1}{k^{2} \beta} \rho_{k}^{2}\right)
$$

Comparing to (2.3) we read off the masses of the winding states:

$$
M_{k}^{2}(\beta \rightarrow 0)=\frac{2 g^{2} N}{\pi}(-1)^{k}
$$

We see that, since the odd $k$ winding states are tachyonic, the confining phase is unstable at high temperature, and deconfinement must occur long before (2.15) is reached. As discussed in [12], (2.15) should be thought of as an analytic continuation of the confining phase into the plasma phase, similar to those routinely performed in string theory. Other comments:

1) Eq. (2.15) should be compared to Polchinski's result for pure four dimensional gauge theory:

$$
M_{k}^{2}(\beta \rightarrow 0)=-\frac{2 g^{2} N}{\pi^{2} \beta^{2} k^{2}}
$$

Both imply deconfinement, but (2.15) seems (at least naively) more like what one would expect in unified string theory. There, one has generically:

$$
\alpha^{\prime} M_{k}^{2}=-C+\beta^{2} k^{2}
$$


valid for all $\beta, k$. C is a universal constant which measures the exponential growth of the density of states. While (2.17) looks more like 2.15) than (2.16) (approaching a universal constant as $\beta \rightarrow 0)$, at finite $\beta$ (2.15) and (2.17) probably differ significantly, at least for generic $m$ for which (2.8) ( $K_{1}$ below is a modified Bessel function):

$$
M_{k}^{2}(\beta)=\frac{2 g^{2} N m k \beta}{\pi}(-1)^{k} K_{1}(m k \beta)+O\left(g^{4}\right)
$$

2) The $(-)^{k}$ in (2.15) looks at first sight embarrassing. It is not clear why, physically, states with even winding in (2.2) should behave differently than those with odd winding. We don't have a good physical interpretation of this sign, but would like to point out that a similar phenomenon occurs in string theory.

Indeed, the origin of the $(-)^{k}$ in 2.15 is the antiperiodic boundary conditions imposed on the fermions (2.1). In superstring theory, precisely the same antiperiodicity leads to a similar strong even-odd difference. One way of seeing that is to notice that antiperiodicity of space-time fermions and modular invariance lead to the fact that the GSO projection imposed for even winding $k$, is the same as in the zero temperature theory (in particular projecting out all tachyons), while for odd $k$ it is opposite to that of the zero temperature theory, allowing tachyons above the Hagedorn transition [11]. Therefore the constant $C$ in (2.17) actually depends on the parity of $k$ for superstring models. It is positive for odd $k$, and nonpositive for even $k$. Another, closely related way of arriving at the same conclusion is to write the vertex operator of a space-time fermion momentum state satisfying (2.1), and show that odd winding Neveu-Schwarz (bosonic) states are mutually local with respect to it, whereas even winding states are not, and have to be modified [13]. The advantage of the second derivation is that it does not use modular invariance, which may or may not hold for the QCD string (see below).

Thus, we see that the $(-)^{k}$ in $(2.15)$ is consistent with a description of the model in terms of a NSR string (with a chiral GSO projection). In fact, the universality of the difference between even and odd winding states in string theory would have been a strong argument against a string interpretation had we not found the above effect in (2.15).

3) We should mention the results of a similar analysis applied to other models of $2 d$ QCD. For pure QCD one finds as $\beta \rightarrow 0: M_{k}^{2}=0$. Hence, there is no instability; the confining phase persists at all temperatures. Models with (any number $N_{f}$ of ) fundamental representation fermions give instead of (2.14):

$$
\mathcal{S}_{\text {fund }}=\frac{N^{2}}{\beta g^{2} N} \sum_{k \neq 0} \frac{1}{k^{2}} \int d x\left(\left(\rho_{k}^{\prime}\right)^{2}+(-)^{k} \frac{2 N_{f}}{\pi N} g^{2} N \rho_{k}\right)
$$


Here too, the confining phase is stable for all temperatures. For bosonic adjoint matter one finds $M_{k}^{2}(\beta \rightarrow 0)=-\frac{2}{\pi} g^{2} N$; it too exhibits a deconfinement transition. This model contains no space-time fermions and the lack of the $(-)^{k}$ compared to (2.15) is consistent with a bosonic string theory (or e.g. a NSR string with a non-chiral GSO projection).

4) The deconfinement transition found for adjoint matter is an indication of an exponentially rising density of single particle states at high energy. We will verify this expectation explicitly below. For the other models mentioned in 3), which confine at all temperatures, the single particle bound state spectrum is known, and it indeed does not exhibit an exponential growth in the density of states with energy.

\section{Supersymmetry in $2 d$ QCD}

The simple calculations in the previous section provide us with some useful qualitative information about the different models, but to study the bound state dynamics, a more detailed analysis is needed. We will review the formalism in this section, whose main purpose is to establish the notation and to discuss a certain accidental supersymmetry which appears in the adjoint model.

It is convenient to use light cone quantization [1], [15], which has been recently applied to this model in [9]. From now on we denote by $\psi_{a b}$ the right moving fermions and by $\bar{\psi}_{a b}$ the left moving ones. The $S U(N)$ currents, $J_{a b}^{+}=\psi_{a c} \psi_{c b}, J_{a b}^{-}=\bar{\psi}_{a c} \bar{\psi}_{c b}$ form right and left moving level $N$ affine Lie algebras, respectively. In the gauge $A_{-}^{a b}=0$ the Lagrangian (11.1) takes the form:

$$
\mathcal{L}=\frac{1}{g^{2}}\left(\partial_{-} A_{+}\right)^{2}+i \psi \partial_{+} \psi+i \bar{\psi} \partial_{-} \bar{\psi}-2 i m \bar{\psi} \psi+A_{+} J^{+}
$$

The equations of motion for $A_{+}, \bar{\psi}$ do not involve derivatives with respect to $x^{+}$, the light cone "time"; it is easy to integrate them out to obtain an action solely in terms of the right moving fermions $\psi$ :

$$
\mathcal{L}_{\psi}=i \psi \partial_{+} \psi+g^{2} J^{+} \frac{1}{\partial_{-}^{2}} J^{+}+i m^{2} \psi \frac{1}{\partial_{-}} \psi
$$

Quantization on constant $x^{+}$surfaces gives rise to the momentum operator:

$$
\begin{aligned}
& P^{+}=i \int d x^{-} \psi \partial_{-} \psi \\
& P^{-}=\int d x^{-}\left(i m^{2} \psi \frac{1}{\partial_{-}} \psi-g^{2} J^{+} \frac{1}{\partial_{-}^{2}} J^{+}\right)
\end{aligned}
$$


Expanding $\psi\left(x^{+}=0\right)$ in modes:

$$
\psi_{a b}\left(x^{-}\right)=\frac{1}{2 \sqrt{\pi}} \int_{-\infty}^{\infty} d k \psi_{a b}(k) e^{-i k x^{-}}
$$

and imposing the canonical anticommutation relation, $\left\{\psi_{a b}\left(x^{-}\right), \psi_{c d}(0)\right\}=\frac{1}{2} \delta\left(x^{-}\right) \delta_{a, d} \delta_{c, b}$ we find the mode anticommutation relations:

$$
\left\{\psi_{a b}(k), \psi_{c d}\left(k^{\prime}\right)\right\}=\delta\left(k+k^{\prime}\right) \delta_{a, d} \delta_{c, b}
$$

$\psi_{a b}(k)$ with $k \leq 0$ are creation operators, whereas the ones with $k \geq 0$ are annihilation operators. The light cone vacuum is chosen such that:

$$
\psi_{a b}(k)|0\rangle=0 \quad \forall k \geq 0
$$

The momentum operators (3.3) are normal ordered in the standard fashion, and take the form:

$$
\begin{aligned}
& P^{+}=\int_{0}^{\infty} d k k \psi_{a b}(-k) \psi_{b a}(k) \\
& P^{-}=m^{2} \int_{0}^{\infty} \frac{d k}{k} \psi_{a b}(-k) \psi_{b a}(k)+g^{2} \int_{0}^{\infty} \frac{d k}{k^{2}} J_{a b}^{+}(-k) J_{b a}^{+}(k)
\end{aligned}
$$

where $J_{a b}^{+}(k)$ is given by (for $\left.k \neq 0\right)$ :

$$
J_{a b}^{+}(k)=\int_{-\infty}^{\infty} d p \psi_{a c}(p) \psi_{c b}(k-p)
$$

$J_{a b}^{+}(0)=\int_{0}^{\infty} d p \psi_{a c}(-p) \psi_{c b}(p)$ must annihilate all physical states (due to confinement).

Physical states are obtained by acting with raising operators $\psi_{a b}(-k)$ on the vacuum (3.6). $P^{+}$is diagonal in this basis; therefore, eigenmodes of $P^{-}$are also eigenmodes of the mass operator, $M^{2} \equiv 2 P^{+} P^{-}$. In the next section we will address the problem of diagonalizing $P^{-}$, but before getting to that it is perhaps useful to point out an interesting property of this system, namely its supersymmetry. The original action (1.1) doesn't appear to be supersymmetric. In terms of on shell degrees of freedom, there are no bosonic ones (no gluons in two dimensions), while the fermionic sector contains $N^{2}-1$ left and right moving degrees of freedom. Consider first the special case $g=m=0$. Then we have a conformal field theory of fermions in the adjoint representation of $S U(N)$; this system is well known to be superconformal. The supersymmetry generator is 4 :

$$
G_{0}=\frac{1}{3} \int d x^{-} \psi_{a b} \psi_{b c} \psi_{c a}
$$

4 This is a special case of the appearance of superconformal symmetry for free fermions $\psi_{i}$ in the adjoint representation of any group. The supercurrent is in general $G(z)=f^{i j k} \psi_{i} \psi_{j} \psi_{k}$, where $f^{i j k}$ are the structure constants of the appropriate Lie algebra. 
The supersymmetry transformation:

$$
\begin{aligned}
\delta \psi_{a b}(p) & =\epsilon\left\{G_{0}, \psi_{a b}(p)\right\}=\epsilon J_{a b}(p) \\
\delta J_{a b}(p) & =\epsilon\left[G_{0}, J_{a b}(p)\right]=-\epsilon N p \psi_{a b}(p)
\end{aligned}
$$

acts non-linearly on $\psi$. In general, adding a mass term and/or gauge interaction destroys the symmetry (3.10). But a look back at (3.2) reveals that for a particular value of $\mathrm{m} / \mathrm{g}$ the interacting Lagrangian is symmetric under (3.10). Indeed, using the (anti) commutation relations 3.10 it is easy to verify that:

$$
\left[G_{0}, P^{-}\right]=0, \text { for } m^{2}=g^{2} N
$$

As is standard in conformal field theory, we also have:

$$
G_{0}^{2}=N P^{+}
$$

Hence, $G_{0}$ commutes with $P^{ \pm}$and therefore with the mass operator $M^{2}=2 P^{+} P^{-}$. For any state $\mid$ phys $\rangle$ with mass $M, G_{0} \mid$ phys $\rangle$ is a state of the same mass and the same value of $P^{ \pm}$, but opposite statistics. Note that $G_{0} \mid$ phys $\rangle$ can not vanish since by (3.12) $G_{0}^{2} \mid$ phys $\rangle=N p^{+} \mid$phys $\rangle$which can never vanish if $M \neq 0$. The physical states are exactly paired by the interaction for the above value of $\mathrm{g} / \mathrm{m}$.

There is one subtlety in the above discussion that needs to be addressed5: supersymmetry could be spontaneously broken in this model, since the Witten index vanishes. Indeed, consider the $g=m=0$ theory on a cylinder with periodic boundary conditions for $\psi_{a b}$. The central charge of the theory is $C=\frac{1}{2}\left(N^{2}-1\right)$, whereas the dimension of the (Ramond) ground state is $\Delta_{0}=\frac{1}{16}\left(N^{2}-1\right)$; since $\Delta_{0}>C / 24, \operatorname{Tr}(-)^{F}=0$. Thus, even when the interaction Lagrangian in (3.2) is exactly supersymmetric, in general supersymmetry can be spontaneously broken. There are many examples where this is known to occur [16]. When we discuss the bound states of the Hamiltonian (3.3) below we will return to this issue and show that supersymmetry is in fact not spontaneously broken in our model (of course, for generic values of $\mathrm{g} / \mathrm{m}$ it is explicitly broken). Qualitatively, this is because the theory is massive, so a Goldstone fermion can not appear.

\footnotetext{
${ }^{5}$ I thank E. Martinec for discussions on this issue.
} 
It is interesting to compare the softly broken supersymmetry of the adjoint QCD model to string expectations. In [6] it is shown that any infrared stable string theory has the following property: the quantity

$$
f(s)=s \sum_{n}(-)^{F_{n}} \int d^{d} p \exp \left[-s\left(p^{2}+M_{n}^{2}\right)\right]
$$

where the sum runs over the spectrum of single particle states with masses $M_{n}$ and fermion numbers $F_{n}$ (which are free as $g_{\mathrm{st}} \rightarrow 0$ ), must satisfy the relation:

$$
\lim _{s \rightarrow 0} f(s)=\text { finite }
$$

Eq. (3.14) is roughly equivalent to the statement that IR stable string theories must have the number of states of a two dimensional theory with a finite number of fields [6]. In string theory this is achieved by quite remarkable cancellations between the contributions to (3.13) of bosons and fermions. There are very few assumptions that go into the proof of (3.14), the main non-trivial one being modular invariance. As mentioned in the introduction, this universality makes it interesting to examine the issue in gauge theory in general, and in our example in particular.

At first glance it seems that in discussing a two dimensional field theory such as $2 d$ QCD, there is not much content in (3.14). That is not the case, because of the very rich spectrum of states that will be discussed in the next section. We will find $\simeq \exp (b \sqrt{n})$ (with a certain constant $b$ ) states with $M^{2} \simeq n$, making $f(s \rightarrow 0)$ a difference of two highly divergent quantities: $f_{B}(s) \sim \sum_{n} e^{b \sqrt{n}-s n} \sim \exp \left(\frac{b^{2}}{4 s}\right)$, and similarly for $f_{F}(s)$. Of course, for $m^{2}=g^{2} N$ the spectrum is exactly supersymmetric, and $f(s)=f_{B}(s)-f_{F}(s)=0$ for all $s$. What is more interesting is that for generic adjoint quark mass $m$, when supersymmetry is explicitly broken, (3.14) still may be satisfied. This is because the soft SUSY breaking in (3.2) splits the low lying states, but asymptotically, at high mass levels, SUSY is effectively restored. It is interesting that a similar asymptotic SUSY is precisely what is required in general for infrared stability in string theory.

Finally, note that the SUSY we found is a symmetry of the gauge fixed Lagrangian (3.2), hence it is respected by interactions, and even non- perturbative effects (in $1 / N$ ), since it holds for any $N$. 


\section{The asymptotic spectrum of bound states}

We turn now to a discussion of the eigenvalue problem for the light cone Hamiltonian (3.7). The analysis of section 2 suggests an exponentially growing density of states $\rho(E) \sim$ $E^{a} e^{b E}$, and our main task in this section is to verify this. Again, we will only outline the derivation of the main results, postponing details to [13].

First, it is convenient to rewrite $P^{-}$(3.7) in terms of fermionic oscillators only, using (3.8). One finds [9]:

$$
\begin{aligned}
& P^{-}=\left(m^{2}-2 g^{2} N\right) \int_{0}^{\infty} \frac{d k}{k} \psi_{a b}(-k) \psi_{b a}(k)+ \\
& g^{2} \int_{0}^{\infty} d k_{1} \cdots d k_{4}\left\{A\left(k_{i}\right) \delta\left(k_{1}+k_{2}-k_{3}-k_{4}\right) \psi_{a b}\left(-k_{3}\right) \psi_{b c}\left(-k_{4}\right) \psi_{c d}\left(k_{1}\right) \psi_{d a}\left(k_{2}\right)+\right. \\
& B\left(k_{i}\right) \delta\left(k_{1}+k_{2}+k_{3}-k_{4}\right)\left[\psi_{a b}\left(-k_{4}\right) \psi_{b c}\left(k_{3}\right) \psi_{c d}\left(k_{2}\right) \psi_{d a}\left(k_{1}\right)+\right. \\
& \left.\left.\psi_{a b}\left(-k_{1}\right) \psi_{b c}\left(-k_{2}\right) \psi_{c d}\left(-k_{3}\right) \psi_{d a}\left(k_{4}\right)\right]\right\}
\end{aligned}
$$

where

$$
A\left(k_{i}\right)=\frac{1}{\left(k_{1}+k_{2}\right)^{2}}-\frac{1}{\left(k_{1}-k_{4}\right)^{2}} ; \quad B\left(k_{i}\right)=\frac{1}{\left(k_{1}+k_{2}\right)^{2}}-\frac{1}{\left(k_{2}+k_{3}\right)^{2}}
$$

A general wave function of a (single string) bosonic bound state is:

$$
\begin{aligned}
\Psi_{B} & =\sum_{n=1}^{\infty} \Psi_{2 n} \\
\Psi_{n} & =\int_{0}^{1} \cdots \int_{0}^{1} d x_{1} \cdots d x_{n} \delta\left(1-\sum_{i=1}^{n} x_{i}\right) \phi_{n}\left(x_{1}, \cdots, x_{n}\right) \operatorname{Tr}\left[\psi\left(-x_{1}\right) \cdots \psi\left(-x_{n}\right)\right]|0\rangle
\end{aligned}
$$

The total $p^{+}$of (4.3) has been set to 1 by Lorentz invariance. The extension to fermionic bound states is obvious. On general grounds [ [ $]$ ], one chooses the "boundary conditions" for the functions $\phi_{n}\left(x_{1}, \cdots, x_{n}\right)$ (which weigh different distributions of the total momentum $p^{+}$between the $n$ constituent quarks):

$$
\phi_{n}\left(0, x_{2}, \cdots, x_{n}\right)=0
$$

Note that since the fermions $\psi$ are real, there is a permutation symmetry of $\phi_{n}$ :

$$
\phi_{n}\left(x_{1}, \cdots, x_{n}\right)=(-1)^{n-1} \phi_{n}\left(x_{2}, \cdots, x_{n}, x_{1}\right)
$$

At large $N, P^{-}$(4.1) operates within the space $\Psi_{B}$ (there is no string splitting). Nevertheless, the problem of diagonalizing $P^{-}$,

$$
P^{-} \Psi_{B}=\lambda \Psi_{B}
$$


is much more formidable than in the 't Hooft model. The main complication is due to the terms in $P^{-}$which change fermion number. These couple different $n$ 's in (4.3). As a first step, we would like in this section to find the asymptotic spectrum of states at high excitation levels. This is interesting for comparison with section 2 and for developing a string description of the theory.

Recall the 't Hooft model [8]: the spectrum of masses (squared) is given by the eigenvalues $\lambda$ of the integral equation,

$$
m_{q}^{2}\left(\frac{1}{x}+\frac{1}{1-x}\right) \phi(x)-\int_{0}^{1} \frac{d y}{(x-y)^{2}} \phi(y)=\lambda \phi(x)
$$

where $m_{q}$ is the renormalized mass of the quark. A general analytic solution of (4.7) is unknown, but at large $\lambda$ things simplify; the integral is dominated by the region $x \simeq y$, corresponding to the long range Coulomb force, and the contribution of the mass term is negligible compared to the binding energy. Thus one can replace (4.7) by:

$$
-\int_{-\infty}^{\infty} \frac{d y}{(x-y)^{2}} \phi(y)=\lambda \phi(x)
$$

The eigenfunctions of (4.8) are $\phi(x)=\sin p x$, with $\lambda=\pi p$. The boundary conditions $\phi(x=0)=\phi(x=1)=0$ pick out $p=n \pi$. The asymptotic spectrum is $\lambda_{n} \simeq \pi^{2} n$.

It is not difficult to repeat the above considerations for the case of adjoint matter. For highly excited states $\Psi_{B}$ in (4.6) (it is clearly enough to consider bosonic bound states because of the asymptotic SUSY of section 3), the action of $P^{-}$on $\Psi_{B}$ is dominated by singular terms in $P^{-}$, corresponding to long range Coulomb forces. Thus the mass term in (4.1) can be neglected; most of the mass of the bound states is due to the binding energy. Furthermore, since only $A\left(k_{i}\right)$ in (4.1) contains a singular term, while $B\left(k_{i}\right)$ is regular (see (4.2)), for highly excited states we should be able to drop the terms which produce and annihilate pairs; we will return to their role below. The operator one needs to diagonalize then is:

$$
P_{c}^{-}=-g^{2} \int d k_{1} \cdots d k_{4} \frac{1}{\left(k_{1}-k_{4}\right)^{2}} \delta\left(k_{1}+k_{2}-k_{3}-k_{4}\right) \psi_{a b}\left(-k_{3}\right) \psi_{b c}\left(-k_{4}\right) \psi_{c d}\left(k_{1}\right) \psi_{d a}\left(k_{2}\right)
$$

which is of interest in its own right, since it summarizes the effect of the $1+1$ dimensional (confining) Coulomb interaction. $P_{c}^{-}$acts diagonally on the $\Psi_{n}(4.3)$. An easy calculation shows that (4.6) with (4.9) substituted for $P^{-}$gives rise to the integral equation:

$$
\frac{\lambda}{g^{2} N} \phi_{n}\left(x_{1}, \cdots, x_{n}\right)=-\int_{-\infty}^{\infty} \frac{d y_{1}}{\left(x_{1}-y_{1}\right)^{2}} \phi_{n}\left(y_{1}, x_{1}+x_{2}-y_{1}, \cdots, x_{n}\right) \pm \text { cyclic permutations }
$$


The right hand side is a sum of $n$ cyclic permutations of $\left(x_{1}, \cdots, x_{n}\right)$ with a $(-1)^{n-1}$ for each elementary cyclic permutation (because of $(4.5)$ ).

Eq. (4.10), which is the analog of (4.8) for our case, is significantly simpler to solve than the original (4.6). One can explicitly find the eigenvalues and eigenmodes $\phi_{n}$. The general analysis is still quite involved, therefore here we will write down explicitly the wave functions $\phi_{n}$ for the first three non-trivial cases, and state the general result for the eigenvalues, leaving the details to [13]. The first non-trivial case is $n=2$ (approximately, a bound state of two adjoint quarks). The wave function $\phi_{2}$ satisfies (4.10):

$$
\frac{\lambda}{2 g^{2} N} \phi_{2}\left(x_{1}\right)=-\int \frac{d y_{1}}{\left(x_{1}-y_{1}\right)^{2}} \phi_{2}\left(y_{1}\right)
$$

Since (4.5) $\phi_{2}(x)=-\phi_{2}(1-x)$, the solution which respects the boundary conditions (4.4) is:

$$
\phi_{2}(x)=\sin \pi n_{1} x ; \quad \lambda_{n_{1}}=2 g^{2} N \pi^{2} n_{1} ; \quad n_{1} \in 2 \mathbf{Z} ; \quad n_{1}>>1
$$

Consider next $n=4$ (bound states of four adjoint quarks). There are many solutions of (4.5), (4.10), but the only one satisfying the boundary conditions is:

$$
\begin{aligned}
& \phi_{4}\left(x_{1}, \cdots, x_{4}\right)=\sin \pi n_{1}\left(x_{1}+x_{2}\right) \sin \pi n_{2}\left(x_{2}+x_{3}\right) \\
& -\sin \pi n_{1} x_{1} \sin \pi n_{2} x_{3}+\sin \pi n_{1} x_{2} \sin \pi n_{2} x_{4}+\left(n_{1} \leftrightarrow n_{2}\right)
\end{aligned}
$$

where $n_{1}, n_{2}$ are even integers. This has eigenvalue (4.6):

$$
\lambda_{n_{1}, n_{2}}=2 g^{2} N \pi^{2}\left(n_{1}+n_{2}\right) ; \quad n_{1}, n_{2} \in 2 \mathbf{Z} ; \quad n_{1}+n_{2}>>2
$$

It is instructive to verify $(4.4),(4.5),(4.10)$ on 4.13 ) (one must remember to impose $\left.\sum x_{i}=1\right)$. Note that the wave function is symmetric under interchange of $n_{1}, n_{2}$; there is one state for each pair $n_{1}>n_{2}$. $\phi_{4}$ (4.13) vanishes when $n_{1}=n_{2}$. 
As our final example we state without proof the result for six quark states $(n=6)$.

$$
\begin{aligned}
& \phi_{6}\left(x_{1}, \cdots, x_{6}\right)=\sin \pi n_{1}\left(x_{1}+x_{2}+x_{3}\right) \sin \pi n_{2}\left(x_{2}+x_{3}+x_{4}\right) \sin \pi n_{3}\left(x_{3}+x_{4}+x_{5}\right) \\
& +\sin \pi n_{1}\left(x_{6}+x_{1}\right) \sin \pi n_{2}\left(x_{1}+x_{2}+x_{3}\right) \sin \pi n_{3}\left(x_{3}+x_{4}\right) \\
& -\sin \pi n_{1}\left(x_{1}+x_{2}\right) \sin \pi n_{2}\left(x_{2}+x_{3}+x_{4}\right) \sin \pi n_{3}\left(x_{4}+x_{5}\right) \\
& +\sin \pi n_{1}\left(x_{2}+x_{3}\right) \sin \pi n_{2}\left(x_{3}+x_{4}+x_{5}\right) \sin \pi n_{3}\left(x_{5}+x_{6}\right) \\
& +\sin \pi n_{1}\left(x_{1}+x_{2}\right) \sin \pi n_{2}\left(x_{2}+x_{3}\right) \sin \pi n_{3} x_{5} \\
& -\sin \pi n_{1}\left(x_{2}+x_{3}\right) \sin \pi n_{2}\left(x_{3}+x_{4}\right) \sin \pi n_{3} x_{6} \\
& +\sin \pi n_{1}\left(x_{3}+x_{4}\right) \sin \pi n_{2}\left(x_{4}+x_{5}\right) \sin \pi n_{3} x_{1} \\
& -\sin \pi n_{1}\left(x_{4}+x_{5}\right) \sin \pi n_{2}\left(x_{5}+x_{6}\right) \sin \pi n_{3} x_{2} \\
& +\sin \pi n_{1}\left(x_{5}+x_{6}\right) \sin \pi n_{2}\left(x_{6}+x_{1}\right) \sin \pi n_{3} x_{3} \\
& -\sin \pi n_{1}\left(x_{6}+x_{1}\right) \sin \pi n_{2}\left(x_{1}+x_{2}\right) \sin \pi n_{3} x_{4} \\
& -\sin \pi n_{1} x_{1} \sin \pi n_{2} x_{3} \sin \pi n_{3} x_{5}+\sin \pi n_{1} x_{2} \sin \pi n_{2} x_{4} \sin \pi n_{3} x_{6} \\
& -\sin \pi n_{1}\left(x_{1}+x_{2}+x_{3}\right) \sin \pi n_{2} x_{2} \sin \pi n_{3} x_{5}+\sin \pi n_{1}\left(x_{2}+x_{3}+x_{4}\right) \sin \pi n_{2} x_{3} \sin \pi n_{3} x_{6} \\
& -\sin \pi n_{1}\left(x_{3}+x_{4}+x_{5}\right) \sin \pi n_{2} x_{4} \sin \pi n_{3} x_{1}+\text { permutations of }\left(n_{1}, n_{2}, n_{3}\right)
\end{aligned}
$$

$n_{1}, n_{2}, n_{3}$ must again be even integers to ensure (4.4), (4.5), (4.10). There is one state (whose wave function is (4.15) ) for each choice of $n_{1}>n_{2}>n_{3}\left(\phi_{6}\right.$ vanishes when any two $n_{i}$ coincide). The eigenvalue (4.6) is,

$$
\lambda_{n_{1}, n_{2}, n_{3}}=2 g^{2} N \pi^{2}\left(n_{1}+n_{2}+n_{3}\right) ; n_{i} \in 2 \mathbf{Z} ; n_{1}+n_{2}+n_{3}>>3
$$

The pattern of (4.12), (4.14), (4.16) continues for higher $n$ as well6. For given $n=2 k$ there is one state satisfying the boundary conditions (4.4) for each choice of $n_{1}>n_{2}>\cdots>n_{k}$, with the spectrum:

$$
M_{n_{1}, \cdots, n_{k}}^{2}=4 g^{2} N \pi^{2}\left(n_{1}+n_{2}+\cdots+n_{k}\right) ; \quad n_{i} \in 2 \mathbf{Z} ; \quad \sum_{1}^{k} n_{i}>>k
$$

The corresponding wave functions $\phi_{2 k}$ vanish whenever $n_{i}=n_{j}$ for any $1 \leq i<j \leq k$. This can be conveniently summarized in a string motivated parametrization: $n_{1}=l_{1}+\cdots+l_{k}$, $n_{2}=l_{2}+\cdots+l_{k}, \cdots, n_{k}=l_{k}$. We find non-trivial states only when all "excitation numbers" $l_{i}>0,(i=1, \cdots, k)$.

${ }^{6}$ I am grateful to S. Shenker for important discussions on this matter. 
Of course, (4.17) provides direct confirmation of an exponentially growing density of states 1 , and the existence of a Hagedorn transition, which was obtained indirectly in section 2 from a different point of view.

So far we have concentrated on the Coulomb part of the light cone Hamiltonian (4.9) finding a rather rich spectrum of states. Two natural questions arise at this stage; do the wave functions $\phi_{2 k}$ corresponding to the spectrum (4.17) form a complete set, and how do the particle creation and annihilation terms in (4.1) modify the spectrum? To discuss these questions, it is useful to introduce a $Z_{2}$ symmetry of the theory, $T$ :

$$
T: \psi_{a b} \rightarrow \psi_{b a}
$$

The Hamiltonian $P^{-}$, (4.1) is invariant under $T$; hence its eigenstates have definite parity under the action of $T$. The wavefunctions $\phi_{2 k}$ constructed above can be shown to satisfy $T \phi_{2 k}=(-1)^{k+1} \phi_{2 k}$. This is related to their antisymmetry under reflection:

$$
\phi_{2 k}\left(x_{1}, x_{2}, \cdots, x_{2 k}\right)=-\phi_{2 k}\left(x_{2 k}, x_{2 k-1}, \cdots, x_{1}\right)
$$

Thus it appears that we are missing all the eigenstates of $P_{c}^{-}$with the opposite $Z_{2}$ charge, and therefore the set $\left\{\phi_{2 k}\right\}$ is incomplete. We have been unable to find any eigenfunctions $\tilde{\phi}_{2 k}$ (of $P_{c}^{-}$) with $T=(-1)^{k}$ which satisfy all the constraints (in particular the boundary conditions), but it is certainly possible that there are additional bound states in the theory to the ones exhibited above; e.g. one may try to relax the boundary conditions; we will return to this issue in [13]. Note that the role of particle creation and annihilation can only be studied once the sector of the theory with $T=(-1)^{k}$ is understood; the mixing between our $\phi_{2 k}$ due to pair creation vanishes $\left.⿴ 囗 ⿱ 一 一\right)$, because of the $Z_{2}$ symmetry: $\left\langle\phi_{2 k}\left|P^{-}\right| \phi_{2 k \pm 2}\right\rangle=0$. Physically, the hope is that one can absorb the effects of pair production in a renormalization of the quarks $\psi_{a b}$. For highly excited states the only interaction between the renormalized quarks should be the Coulomb interaction (4.9).

\footnotetext{
7 The generating functional is $\operatorname{Tr} e^{-s M^{2}} \simeq \prod_{n=1}^{\infty}\left(1+e^{-s n}\right)$.

8 This is in sharp contrast to what happens for bosonic adjoint matter.
} 


\section{Supersymmetry breaking and the extreme infrared theory}

In the previous section we have concentrated on the asymptotic behavior of the spectrum in the bosonic sector. Assuming SUSY is not spontaneously broken (for $m^{2}=g^{2} N$ ), the spectrum of fermionic bound states is the same. It is time to present a proof of that assumption. We will actually give two arguments for this result, the first of which is the following. Consider a bound state $\Psi_{B}(4.3)$. The fermionic state related to it by SUSY is $G_{0} \Psi_{B}$. We have to verify that this state is a physical bound state. Clearly, $G_{0} \Psi_{B}$ is an eigenstate of $P^{-}$; hence, it is enough to check that it satisfies the boundary conditions (4.4). A straightforward calculation [13] reveals that this is indeed the case. As a simple example, consider:

$$
G_{0} \int_{0}^{1} d x \phi_{2}(x) \operatorname{Tr}[\psi(-x) \psi(x-1)]|0\rangle=2 \int_{0}^{1} d x \int_{0}^{x} d y \phi_{2}(x) \operatorname{Tr}[\psi(-y) \psi(y-x) \psi(x-1)]|0\rangle
$$

This has the form (4.3) with $\phi_{3}\left(x_{i}\right)=-2 \phi_{2}\left(x_{1}\right)-2 \phi_{2}\left(x_{2}\right)-2 \phi_{2}\left(x_{3}\right)$, which indeed vanishes for $x_{1}=0$, because of the antisymmetry and boundary conditions satisfied by $\phi_{2}$. This generalizes for all $n$. Hence $G_{0} \Psi_{B}$ is physical and SUSY is unbroken.

A second way to show that for $m^{2}=g^{2} N$ SUSY is not spontaneously broken is to establish the absence of massless fermions in the spectrum. Of course, a massless Goldstone fermion must appear if SUSY is to be spontaneously broken. Since it is clear from (4.1) that the masses of bound states are monotonically increasing functions of the bare quark mass $m$, it is enough to establish this fact for $m=0$.

Consider then (1.1) at $m=0$. At very large distance scales the gauge coupling $g \rightarrow \infty$ and the $F_{\mu \nu}^{2}$ term decouples. The resulting theory is closely related to the Lagrangian construction of coset models (see e.g. [17]) and is quite well understood. It is a conformal field theory with central charge $C_{I R}=C_{U V}-C_{\text {gauge }}$ where $C_{U V}=\frac{1}{2}\left(N^{2}-1\right)$ is the central charge of the free fermion theory, and $C_{\text {gauge }}=\frac{N\left(N^{2}-1\right)}{N+N}=\frac{1}{2}\left(N^{2}-1\right)$ is the amount by which gauging the level $N S U(N)$ affine Lie algebra reduces $C$. Thus we find that in the infrared, $C_{I R}=0$ : there are no massless degrees of freedom at large distances. In

particular there are no massless fermions (which would require $C_{I R} \geq \frac{1}{2}$ ) for $m=0$ and therefore for all $m$. Hence SUSY can not be spontaneously broken.

The infrared theory (for massless adjoint quarks):

$$
\mathcal{L}_{I R}=\bar{\psi} \gamma^{\mu} D_{\mu} \psi
$$


is actually an interesting topological theory in space-time. Although, as we saw, it contains no field theoretic degrees of freedom, the theory contains a rich spectrum of global modes, whose correlation functions are independent of position (since we have taken the distance scale to infinity). There is a well developed technology for studying these states [17]: for each primary operator of the fermion $S U(N)$ Kac-Moody algebra there is one global mode in the gauged theory (5.1) at ghost number zero 9 , obtained by dressing the fermionic operator with gauge fields; $A_{\mu}$ does have global degrees of freedom in $2 d$. The group theory leads to the following result [13]: for each Young diagram $R$ with $n$ boxes, there is one state in the infrared theory, obtained by multiplying $n \psi$ 's (the representations of $S U(N)$ that appear in the adjoint fermion conformal field theory are all representations of the form $R \times \bar{R})$. Since there are $P(n)$ such diagrams, we see that in the limit $N \rightarrow \infty$ one finds a very rich spectrum of global modes.

This is to be compared with a similar calculation in the 't Hooft model, where one finds one state for each completely antisymmetric Young diagram with $n$ boxes. Thus there is one state for each integer $n$. It is amusing that in both cases (of adjoint and fundamental matter) there seems to be a (very) rough correspondence between the spectrum of massive bound states at finite $g$, and that of the global modes in the extreme IR limit (in a string description, the nature of the corresponding states would appear to be quite different).

There has been some recent work on realizing space-time topological theories as target space theories corresponding to topological world sheet theories [18]. Perhaps an understanding of the world sheet description of the extreme IR limit of adjoint QCD would be a useful first step towards a complete string description of the theory (1.1) at all scales.

\section{Concluding Remarks}

The main motivation for this work has been to understand the relation (if any) of confining large $N$ gauge theories with a non-trivial spectrum of bound states and string theory. $2 d$ QCD coupled to adjoint matter is perhaps the simplest theory with an (exponentially) infinite number of Regge trajectories; it is closely related to four dimensional QCD via dimensional reduction, and should provide a useful laboratory for studying the relation of QCD to string theory. Many of our results for this theory will look familiar to string theorists, but (barring an inspired guess) much remains to be done before the correspondence can be made more precise.

9 There are states at other ghost numbers as well; we will not be concerned with those here. 
The asymptotic spectrum of infinitely many linear Regge trajectories looks of course quite stringy. But it is (probably) not exactly linear down to the ground state. Furthermore, it is interesting that the spectrum resembles that of an open string (of course, if at all, this gauge theory is expected to be related to a closed string): there are $n_{i}$ (4.17) but no $\bar{n}_{i}$. In closed superstring theory (a natural candidate would be the three dimensional version of [19]), one would generically find states parametrized by $n_{i}, \bar{n}_{i}$, from left and right movers on the world sheet. It is also interesting that states with "occupation numbers" $l_{i}=0$ don't seem to appear in QCD (4.17). This reduction of the number of states of the "QCD string" compared to a fundamental one may actually be responsible (among other things) for the absence of space-time gravity in the former.

The asymptotic supersymmetry discussed in section 3 may also be a manifestation of a stringy structure, but this issue is far from clear to the author. We haven't discussed in detail the bosonic version of adjoint QCD, but it too has a Hagedorn transition (section 2) and an infinite number of asymptotically linear Regge trajectories [13]. In string theory,

such a system would be unstable in the infrared. The question of whether bosonic adjoint matter coupled to $2 d$ QCD also develops an infrared instability in the $1 / N$ expansion remains open. We hope to return to it in [13].

Other favorable signs include the pattern of masses of winding modes discussed in section 2, and the topological theory in the infrared, which probably has a world sheet interpretation. Possible future steps in the attempt to uncover a string description are a study of the S-matrix of the model, which may provide more hints (like duality, the exact mass spectrum etc), and a direct construction of a string in space-time out of the quarks, which act as small bits of string. And, if the string idea fails, adjoint $2 d$ QCD seems to be a good place to understand why, and find alternative ways to deal with the physics of a weakly interacting system containing an infinite number of linearly rising Regge trajectories. Clearly, a much better understanding of that physics is required to address these issues.

\section{Acknowledgements}

I am grateful to T. Banks, P. Hořava, I. Klebanov, E. Martinec, N. Seiberg and S. Shenker for discussions. This work was partially supported by DOE grant DEFG02-90ER40560 and the L. Block foundation. 


\section{References}

[1] G. 't Hooft, Nucl. Phys. B72 (1974) 461.

[2] E. Witten, Nucl. Phys. B160 (1979) 57; S. Coleman, "Aspects of Symmetry", Cambridge University Press (1985).

[3] A. Polyakov, "Gauge Fields and Strings", Harwood Academic Publishers (1987).

[4] J. Polchinski, Texas preprint UTTG-16-92, hep-th/9210045.

[5] P. Ginsparg and G. Moore, 1992 TASI lectures, hep-th/9304011.

[6] D. Kutasov and N. Seiberg, Nucl. Phys. B358 (1991) 600.

[7] D. Gross, Princeton preprint PUPT-1356 (1992), hep-th/9212149; D. Gross and W. Taylor, preprint PUPT-1376, LBL-33458, hep-th/9301068.

[8] G. 't Hooft, Nucl. Phys. B75 (1974) 461.

[9] S. Dalley and I. Klebanov, Phys. Rev. D47 (1993) 2517.

[10] D. Gross, R. Pisarski and L. Yaffe, Rev. Mod. Phys. 53 (1981) 43.

[11] J. Atick and E. Witten, Nucl. Phys. B310 (1988) 291.

[12] J. Polchinski, Phys. Rev. Lett. 68 (1992) 1267.

[13] D. Kutasov, to appear.

[14] N. Weiss, Phys. Rev. D24 (1981) 475; D25 (1982) 2667.

[15] H. Pauli and S. Brodsky, Phys. Rev. D32 (1985) 1993, 2001; K. Hornbostel, S. Brodsky and H. Pauli, Phys. Rev. D41 (1990) 3814.

[16] E. Witten, Nucl. Phys. B202 (1982) 253.

[17] K. Bardacki, E. Rabinovici and B. Saring, Nucl. Phys. B299 (1988) 151; K. Gawedzki and A. Kupianen, Nucl. Phys. B320 (1989) 625.

[18] S. Elitzur, A. Forge and E. Rabinovici, Nucl. Phys. B388 (1992) 131.

[19] D. Kutasov and N. Seiberg, Phys. Lett. 251B (1990) 67. 